Validity of the Negative Binomial Distribution in Particle Production

This article has been downloaded from IOPscience. Please scroll down to see the full text article. 1987 Europhys. Lett. 41127

(http://iopscience.iop.org/0295-5075/4/10/008)

View the table of contents for this issue, or go to the journal homepage for more

Download details:

IP Address: 139.165.107.21

The article was downloaded on 05/12/2011 at 15:56

Please note that terms and conditions apply. 
Europhys. Lett., 4 (10), pp. 1127-1131 (1987)

\title{
Validity of the Negative Binomial Distribution in Particle Production.
}

\author{
J. Cugnon $(*)$ and O. Harouna $(* *)$

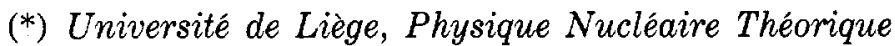 \\ Institut de Physique au Sart Tilman, B. 5, B-4000 Liège 1, Belgique \\ (**) Université de Niamey, Service de Physique \\ B.P. 10662 Niamey, République du Niger
}

(received 27 July 1987; accepted in final form 7 September 1987)

PACS, 25.90. - Other topics in nuclear reactions and scattering: specific reactions.

\begin{abstract}
Some aspects of the clan picture for particle production in nuclear and in highenergy processes are examined. In particular, it is shown that the requirement of having logarithmic distribution for the number of particles within a clan in order to generate a negative binomial should not be taken strictly. Large departures are allowed without distorting too much the negative binomial. The question of the undetected particles is also studied. It is shown that, under reasonable circumstances, the latter do not affect the negative binomial character of the multiplicity distribution.
\end{abstract}

\section{Introduction.}

It is more and more evident [1] that the charged-particle multiplicity distributions in many baryonic or semi-leptonic processes at high energy closely follow a negative binomial (NB) law, both for total multiplicities and for restricted intervals of pseudorapidity. Furthermore, it has been suggested that NB could be relevant for some nuclear processes [2]. Recently, experimental evidence was discovered in low-energy antiproton annihilation on nuclei [3]. It is not yet clear that there is a simple physical explanation for the generality of this law. At least, a necessary, but not sufficient condition, is that conservation laws (energy, baryon number, charge, ...) should not put too strong a constraint on the production process $[3,4]$. The more appealing explanation has been put forward by Van Hove and Giovannini $[4,5]$. They showed that NB arises naturally from a cascade process, where particles are produced in independent bunches (or clans), which means that the number of clans is a Poisson variable, and where the number of particles within a clan follows a logarithmic law. The independence of clans may look natural or, at least, consistent with current models. On the contrary, the logarithmic law seems quite a strong and unusual condition. In this note, we give arguments, which indicate that this condition may be-sometimes considerably-relaxed, without disturbing too much the NB law. We also tackle another aspect of the problem. Quite often, neutral particles are not detected and NB 
is expected to hold for all types of particles. We show that if this is true, this remains the case for charged particles, provided once again the charge conservation law is not influencing the production process.

\section{Approximate conditions for negative binomials.}

Let $x_{1}, \ldots, x_{N}$ be independent stochastic variables of the same logarithmic law and $N$ be a Poisson variable. It is well known that $x=x_{1}+\ldots+x_{N}$ is a stochastic variable with a NB law. This theorem is generally formulated for the generating function. For the NB of parameters $r$ and $p$ (we closely follow here the notation of ref. [6]), the generating function is

$$
g_{\mathrm{NB}}(s)=\left(\frac{p}{1-q s}\right)^{r},
$$

with $q=1-p, 0<p<1$. It can be rewritten as

$$
g_{\mathrm{NB}}(s)=\exp \left[-r \ln p^{-1}(1-g(s))\right],
$$

where

$$
g(s)=g_{\mathrm{L}}(s)=\frac{\ln (1-q s)}{\ln p}
$$

is the generating function for the logarithmic distribution. One can wonder whether the "gross properties» of the NB are preserved when the quantity $g(s)$ is perturbed from value (3). Of course, one has to agree on a convenient definition of the gross properties, whose precise form could depend upon the context of the problem one is looking at. We simply focus here on the first two moments $m_{1}$ and $m_{2}$. There is a rather straightforward relation between $m_{1}$ and $m_{2}$ and $g(s)$. One has

$$
g^{\prime}(1)=\frac{m_{1}}{r \ln p^{-1}}
$$

and

$$
g^{\prime \prime}(1)=\frac{m_{2}-m_{1}-m_{1}^{2}}{r \ln p^{-1}} .
$$

The conclusion is that any distribution

$$
g(s)=g_{\mathrm{L}}(s)+h(s),
$$

with $h(1)=h^{\prime}(1)=h^{\prime \prime}(1)=0$, when composed with the Poisson distribution, will yield a distribution with the first two moments as distribution (2). We do not look at the higher moments systematically. Rather, we numerically investigate the modification of the NB, assuming a plausible modification of $g(s)$. We choose for instance

$$
h(s)=A s(1-s)^{3},
$$

which is the polynomial of the lowest degree $(P(0)=0$ for the logarithmic distribution). The fact that $g(s)$ should be a generating function limits the value of $A$. The maximum positive and negative values of $A$ are given in fig. 1, as a function of $p$. In fig. 2 and 3 , we show the 


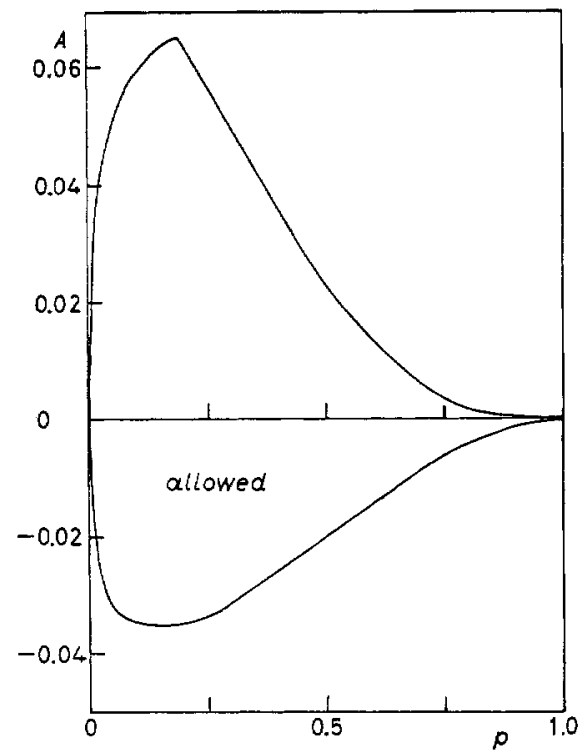

Fig. 1. - Maximum (upper curve) and minimum (lower curve) values of the quantity $A$ entering in eq. (7) as a function of the parameter $p$ of the logarithmic distribution.

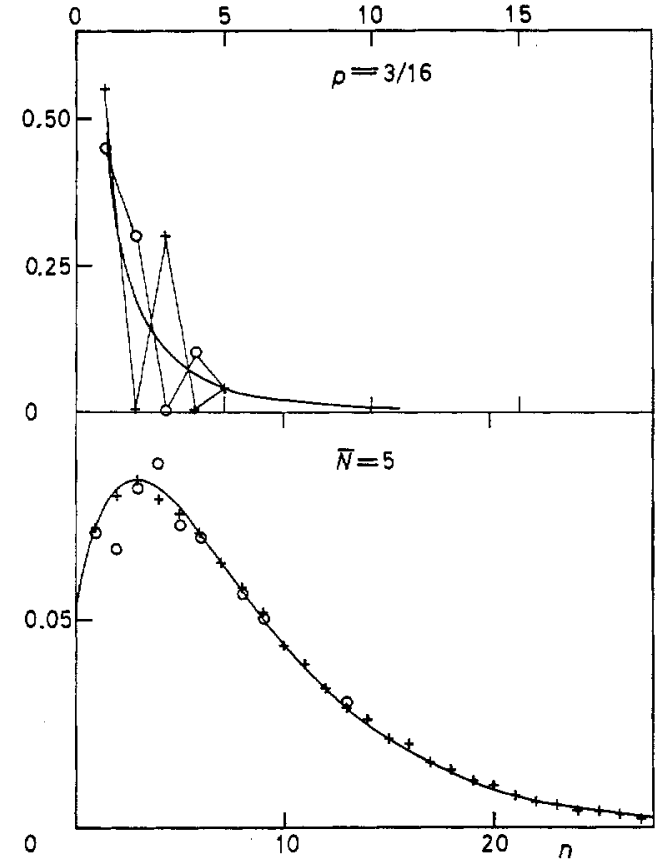

Fig. 2.

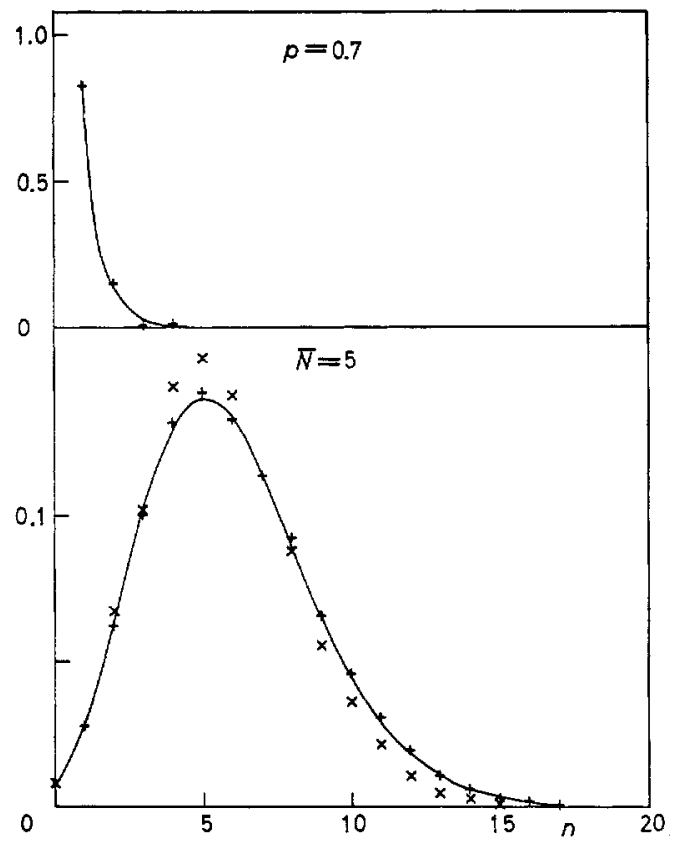

Fig. 3 .

Fig. 2. - Upper part: logarithmic distribution (full curve) for $p=3 / 16$ and its modification using the maximum (crosses) and the minimum (circles) value of $A$ in eq. (7). Lower part: NB distribution (full curve) and modifications induced by the one of the logarithmic distribution: $+A_{\max }, \circ A_{\min }$. See text, for details.

Fig. 3. - Same as fig. 2 for $p=0.7$. The symbols $(x)$ correspond to cutting the tail of the logarithmic distribution beyond $n=2$. 
calculated distribution laws for two typical cases and compare them with the original NB distributions. Figure 2 shows one of our most spectacular results. Despite the strong modification of the logarithmic distribution, there is only a small modification of the NB. Note that higher-order polynomials for $h(s)$ (in eq. (7)) do give smaller perturbations of the logarithmic distribution, essentially in the tail. We also looked at such modifications and observed smaller changes than the ones indicated in the lower part of fig. 2 . We have to stress that, in general, the physical situation is closer to fig. 3 than to fig. 2 , since, often, the average size of the clans is smaller than the number of clans. We also looked at the modification of the Poisson law, which gives rise to changes comparable with those of the logarithmic law, but this aspect of the clan formalism is probably less subject to changes. In conclusion, we are enabled to state a «flexible" theorem, saying that if clans are produced independently, and if the number of particles in a clan decreases (on the average), the multiplicity will follow a NB to a good accuracy. See, f.i., the extreme case of fig. 3, when only $n=1$ and $n=2$ clans are retained.

\section{Charged and neutral particles.}

Ejected particles, in a p-nucleus interaction, f.i., can be charged or neutral. Let us assume that any ejected particle can be charged with a probability $u(0 \leqslant u \leqslant 1)$, independently of the other ones. This is quite reasonable if the multiplicity is low. Then, protons and neutrons for instance can be emitted without restriction and $u$ may be taken as the $Z / A$ ratio. If the multiplicity for all particles follow a NB distribution, does the chargedparticle multiplicity also follow a NB distribution? We show that the answer is yes. This can be done explicitly, but, as usual, the demonstration is trivial in terms of generating functions. Indeed, we deal with a composition of probability distributions [6]. If $g_{\mathrm{NB}}(s)$ is the original function, the composed generating function is

$$
g(s)=g_{\mathrm{NB}}(1-u+u s)
$$

or with the help of eq. (1)

$$
g(s)=\left(\frac{p}{1-q(1-u+u s)}\right)^{r},
$$

which has the standard form for a NB, after the substitution

$$
p \rightarrow \frac{p}{p+q u}, \quad q \rightarrow \frac{q u}{p+q u} .
$$

The mean value $\bar{n}$ is reduced by a factor $u$, of course. Furthermore, one has for the new NB

$$
\frac{\sigma^{2}}{\bar{n}}=1+\frac{q}{p} u,
$$

which means that the dispersion $\sigma$ is proportionally reduced. In terms of the parametrization used in ref. [5], the modification (10) amounts to $\bar{n} \rightarrow \bar{n} u, k \rightarrow k$. Let us notice that the invariance of the probability law after selection is fulfilled for many distributions (logarithmic, geometric, Poisson, f.i.), but is by no means universal. The validity of the NB in restricted rapidity intervals at high energy could be a consequence of the validity of NB for total multiplicity and of the above property. Indeed, if the interval is small enough, one 
can consider that the probability for having a particle in this interval is a constant and does not depend upon the other particles. Of course, nothing indicates that $u$ should be the same for all rapidity intervals.

As an illustration, we reanalysed the $\bar{p}$-emulsion data of ref. [7]. In ref. [3], it is shown that the charged-particle multiplicity fits to a NB with $\bar{n} \approx 4.7$ and $k \approx 6.5$ (at rest). Correcting for neutrons, this gives $\bar{n} \approx 10$. The latter value gives $\sim 6$ clans (instead of $\sim 3.5$ ) on the average with an average number of particles of $\sim 1.7$ (instead of $\sim 1.3$ ). The size of the clans does not change on the whole range of data of ref. [7], extending from rest to

$1400 \mathrm{MeV} / \mathrm{c}$.

\section{REFERENCES}

[1] CARLSON P., Multiplicity Distributions, in Proceedings of the XXII International Conference on High Energy Physics, Berkeley, July 1986, Vol, 2, edited by S. C. LoKEN (World Scientific, Singapore) 1987 , p. 1346.

[2] Cugnon J., Z. Phys. A, 327 (1987) 187.

[3] Cugnon J., Jasselette P. and Vandermeulen J., Europhys. Lett., 4 (1987) 535.

[4] van Hove L. and Grovannini A., Multiparticle Dynamics, in Proceedings of the XVII International Symposium, Seewinkel, Austria, June 1986, edited by M. MARKYTAN et al. (World Scientific, Singapore) 1987 , p. 561.

[5] Giovannini A. and van Hove L., Z. Phys. C, 30 (1986) 391.

[6] FeLLer W., An Introduction to Probability Theory and its Applications, Vol. 1, 3rd ed. (John Wiley, New York, N.Y.) 1968.

[7] BAtusov Y. A. et al., Europhys. Lett., 2 (1986) 115. 